\title{
Transnational social work: Opportunities and challenges of a global profession
}

\author{
Allen Bartley and Liz Beddoe (Eds.) \\ Policy Press, Bristol, UK, 2018 \\ ISBN 978-1447333364, pp. 276, hardback, NZD190.00
}

$\mathrm{T}$ This edited collection considers transnational social work with a particular emphasis on the opportunities and challenges within Aotearoa New Zealand, Australia, the UK, Ireland and Canada. A review of any edited collection does not provide the opportunity to comment on all chapters or necessarily all themes covered in the text. The whim and professional space of the reviewer naturally leads to a greater focus on chapters that discuss conundrums for the reviewer or open windows of opportunity for further research.

A theme running through this book, whether from the perspective of individual social workers, employers or regulators - either professional or statutory - is the challenge of ensuring social workers are properly inducted into the specific culture of the host country. The chapter by Walsh-Tapiata and her colleagues is a delightful read. It presents a way of welcoming and orienting transnational social workers to the specific Aotearoa New Zealand context using pōwhiri through which relationships are established. They emphasise that this initial encounter should take place on a marae to further develop an understanding of Māori connections to place, land and ancestors. It is not apparent that other countries have a similar process.

Papadopoulos, in her chapter on recognising transnational social workers in Australia, mentions briefly the ethical implications of social workers migrating from countries that need their services. This is not an issue for the social work profession only, but certainly one that rarely receives the attention it should. Papadopoulos also discusses the concept of recognition - normally used in a regulatory sense to discuss credentialing or mutual regulation processes. Papadopoulos extends the concept of recognition to the recognition of the individual social worker as ethical practice and required as respect for the cultural capital an individual brings with them. She argues that an ethnocentric approach to recognition may limit the possibilities that transnational social workers can bring to the development of the profession in the host country.

Sansfaçon and colleagues consider the transferability of skills of transnational social workers. Although they found differences in practice between transnational social workers coming to Canada from the Global North and the Global South, these mainly related to interventions rather than assessments. Differences included priorities for intervention, working with families and community and accessing resources. They found that, while it was possible to see how the political and cultural context may influence intervention, there were no significant changes in the values of the transnational social workers. This chapter suggests, to me, that further research is warranted on how context-specific issues are addressed while foundational social work values remain unchanged.

From my perspective as a regulator, I see the challenges worthy of more discussion. 
A particular challenge for transnational social work that needs further research is the assessment of international qualifications. Given the international definition of social work and the guidelines from the IASSW, there is some expectation that all qualifications would have some commonality despite being country-specific. However, should there be minimum core requirement?

Although I am not sure who the target audience is for this publication, it will no doubt appeal to a range of practitioners and employers. I would hope to see regulators - particularly those in the statutory environment - joining the debates that this book will generate. I am also hoping that this is the first of a series, as this book has a focus on migration to, and practice in, Commonwealth countries. It would be interesting to consider the position of transnational social workers in other nations that have a focus on indigenous social work and the consequences for countries that do not retain their social workers. I commend the book. It is a very timely publication that addresses what will be a continuing, and growing, professional issue.

Reviewed by Jan Duke Social Workers Registration Board, NZ 